\title{
Pre-test CFD simulations of the NACIE-UP BFPS test section
}

\author{
R. Marinari ${ }^{1 *}$, I. Di Piazza ${ }^{2}$, N. Forgione ${ }^{1}$, F. Magugliani ${ }^{3}$ \\ ${ }^{1}$ Università di Pisa, DICI, Pisa, Italy \\ ${ }^{2}$ ENEA FSN-ING, C.R. Brasimone, Camugnano (Bo), Italy \\ ${ }^{3}$ Ansaldo Nucleare, Genova, Italy \\ ${ }^{*}$ Corresponding author. E-mail: ranieri.marinari@gmail.com
}

\section{HIGHLIGHTS}

- Numerical study of flow blockage phenomena in a HLM cooled pin bundle adopting ANSYS CFX code;

- The SST- $k$ - $\omega$ turbulence model is adopted;

- CFD results adopted for the correct location of the instrumentation;

- Preliminary analysis for a flow blockage detection system;

\section{KEYWORDS}

Heavy liquid metals; LBE; CFD; fuel pin bundle; flow blockage

\section{Abstract}

The present paper is focused on the CFD pre-test analysis and design of the new experimental facility 'Blocked' Fuel Pin bundle Simulator (BFPS) that will be installed into the NACIE-UP (NAtural CIrculation Experiment-UPgrade) facility located at the ENEA Brasimone Research Center (Italy).

The BFPS test section will carry out suitable experiments to fully investigate different flow blockage regimes in a 19 fuel pin bundle providing experimental data in support of the development of the ALFRED (Advanced Lead-cooled Fast Reactor European Demonstrator) LFR DEMO.

The geometrical domain of the fuel pin bundle simulator was designed to reproduce the geometrical features of ALFRED, e.g. the external wrapper in the active region and the spacer grids. Pre-tests calculations were carried out by applying accurate boundary conditions; the conjugate heat transfer in the clad is also considered.

The blockages investigated are internal blockages of different extensions and in different locations: central sub-channel blockage, corner sub-channel blockage, edge sub-channel blockage, one sector blockage, and two-sector blockage.

RANS simulations were carried out adopting the ANSYS CFX commercial code with the laminar sublayer resolved by the mesh resolution. The loci of the peak temperatures and their width as predicted by the CFD simulations are used for determining the location of the pin bundle instrumentation. The CFD pre-test analysis allowed also investigating the temperature distribution in the clad to operate the test section safely.

\section{Symbols}
$D$
External pin diameter [mm]
$f_{\text {Darcy }}$
$H$
Darcy-Weisbach friction factor [-]
Exhagonal key of the bundle
Lactive
$L_{\text {plenum }}$
Length of the heating part of the pins [mm]
$L_{\text {total }}$
Length of the mixing region downstream of a FA [mm]
$N_{\text {pins }}$
Total length of the pin [mm]
Number of pins per FA [-] 


$\begin{array}{lll}48 & N u & \text { Nusselt number }[-] \\ 49 & P / D & \text { Pitch-to-diameter ratio }[-] \\ 50 & \operatorname{Pr}_{t} & \text { turbulent Prandtl number }[-] \\ 51 & Q & \text { Maximum heating power }[\mathrm{kW}] \\ 52 & Q_{p i n} & \text { Maximum power of a pin }[\mathrm{kW}] \\ 53 & q ” & \text { Maximum het flux }\left[\mathrm{MW} / \mathrm{m}^{2}\right] \\ 54 & r & \text { radial coordinate }[\mathrm{mm}] \\ 55 & \operatorname{Re}_{B F P S} & \text { BFPS Reynolds number }[-] \\ 56 & T_{\text {pin,max }} & \text { Maximum pin temperature }\left[{ }^{\circ} \mathrm{C}\right] \\ 57 & v & \text { Subchannel velocity }[\mathrm{m} / \mathrm{s}] \\ 58 & y^{+} & \text {dimensionless wall distance }[-] \\ 59 & \mathrm{z} & \text { axial coordinate }[\mathrm{mm}]\end{array}$

60 Acronyms

\begin{tabular}{|c|c|}
\hline AISI & American Iron and Steel Institute \\
\hline ALFRED & Advanced Lead Fast Reactor European Demonstrator \\
\hline BI & Blockage Index \\
\hline BFPS & Blocked Fuel Pin bundle Simulator \\
\hline CFD & Computational Fluid Dynamic \\
\hline DEMO & DEMOnstrator \\
\hline ENEA & Agenzia nazionale per le nuove tecnologie, l'energia e lo sviluppo economico sostenibile \\
\hline EU & European Union \\
\hline FA & Fuel Assembly \\
\hline FBR & Fast Breeder Reactor \\
\hline FFT & Fast Fourier Transform \\
\hline FPS & Fuel Pin Simulator \\
\hline FP7 & Framework Program 7 \\
\hline GEN-IV & Generation IV \\
\hline HLM & Heavy Liquid Metal \\
\hline HLMR & Heavy Liquid Metal Reactor \\
\hline HTC & Heat Transfer Coefficient \\
\hline $\mathrm{HX}$ & Heat eXchanger \\
\hline H2020 & Horizon 2020 project \\
\hline KIT & Karlsruher Institute für Technologie \\
\hline LBE & Lead Bismuth Eutectic \\
\hline LEADER & Lead European Advanced Demonstrator Reactor \\
\hline LES & Large Eddy Simulation \\
\hline LFR & Lead Fast Reactor \\
\hline NACIE-UP & NAtural CIrculation Experiment Upgrade \\
\hline O.D. & Outside Diameter \\
\hline PFBR & Prototype Fast Breeder Reactor \\
\hline RANS & Reynolds Average Navier-Stokes \\
\hline SESAME & Thermal-hydraulics Simulations and Experiments for the Safety Assessment of MEtal cooled reactors \\
\hline SST & Shear Stress Transport model \\
\hline TC & ThermoCouple \\
\hline
\end{tabular}

\section{Introduction}

In the context of GEN-IV heavy liquid metal-cooled reactors safety studies, the flow blockage in a fuel sub-assembly is considered one of the main issues to be addressed and one of the most important and realistic accident for Lead Fast Reactors (LFR) fuel assembly. The blockage in a fast reactor Fuel Assembly (FA) may have serious effects on the safety of the plant leading to the FA damaging or melting. The temperature of the coolant leaving the FA is considered an important indicator of the health of the FA (i.e. 
the effective heat removal) and is usually monitored via a dedicated, safety-related system (e.g. thermocouple). The external or internal blockage of the FA may impair the correct cooling of the fuel pins, be the root cause of anomalous heating of the cladding and of the wrapper and potentially impact also fuel pins not directly located above or around the blocked area. In order to model the temperature and velocity field inside a wrapped FA under unblocked and blocked conditions, detailed experimental campaign as well as 3D thermal hydraulic analyses of the FA is required. The root causes of the FA blockage are aggregation of solid matter (oxides), dislodged from its intended location or generated in the coolant, transported inside and along with the coolant's flow; this matter could stop inside the FA (mainly because of its narrow spaces) and interfere with the coolant flowing inside the FA. The main consequence of the blockage is a reduction of the coolant flow rate through the FA. Blockage can be instantaneous (when a large enough piece of material obstructs a portion of the channels of the FA) or time dependent (when the aggregation of solid matter piles up in the channels of the FA). For grid-spaced FAs, an internal blockage is generally located in the first grid and it has a flat-like shape (Schultheiss, 1987). FAs are usually designed with a number of inlets slots to prevent a complete and instantaneous blockage.

Fuel Assembly blockage, total or partial, has been extensively analyzed since the early days of fast reactors. While many of these studies refer to Sodium Fast Reactors, the results may be a starting point for LFRs too. The focus of these analyses is to determine the effects of a blockage on the temperature (cladding and coolant) and pressure (coolant) inside the FA as well as at the outlet of the subassembly, and to find the optimal detection techniques. Investigations carried out within the LEADER FP7 EU project showed that the maximum clad temperature to avoid long-term creep in the Ti15-15 cladding material is $650{ }^{\circ} \mathrm{C}$ (Weisenburger, 2013).

Wey et al. (1982) described the development of a Monte Carlo model capable of generating real time temperature signals from a specified input temperature profile and turbulent velocity field. The model used multi-particle batches, which permit calculations simulating heat dissipation processes. Various Sodium Loop experiments are outlined which were used to validate the model in turbulent pipe and jet flows. For the latter, a multistage technique was used which enabled modeling of axial variation of the turbulence field. The technique has also been used to predict temperature signals at the outlet of subassemblies with blockages of various sizes at center and corner positions. A simple assessment of the viability of detecting such blockages with a single centrally located sensor is presented.

Hae-Yong Jeong et al. (2014) analyzed the temperature rise across each subassembly in order to detect the formation of a significant size of blockage. The blockage detection logic of the Prototype Fast Breeder Reactor (PFBR) generates an alarm signal when the assembly-wide temperature rise deviates $5 \mathrm{~K}$ from the pre-determined value and scram signal for a deviation greater than $10 \mathrm{~K}$. The paper proposed a concept of Blockage Index (BI) by adopting a kernel function of Gaussian type to take advantage of the thermalhydraulic information in neighboring subassemblies. With the blockage index, a distance-weighted temperature changes for each subassembly is defined. Combining the blockage index for each subassembly into a core wide map of blockage indices, useful information are gathered to determine whether any blockage is introduced or not at some location of subassembly. It is also possible to adjust the range of influence by performing sensitivity studies on reference distance in advance to select a distance factor which describes the blockage phenomena most correctly for the given subassembly design.

Seung-Hwan Seong et al. (2006) used the LES turbulence model in the ANSYS CFX code for analyzing the temperature fluctuation in the upper plenum. After analyzing the temperature fluctuations in the upper plenum, a basic design requirement was established for the flow blockage detection system through a FFT analysis and a statistical analysis. They concluded that response time of a measuring device was less than $13 \mathrm{~ms}$ and that it should cover a high temperature range of $1000 \mathrm{~K}$. In addition, the resolution of the thermocouple should be less than $2 \mathrm{~K}$ and its location should be within $25 \mathrm{~cm}$ from the exit of each assembly.

Nomoto et al (1980) installed thermocouple temperature sensors above the central region of the core in the JOYO experimental sodium fast reactor to monitor the outlet coolant temperature of 115 subassemblies. Subassembly outlet coolant temperature distributions were obtained under various power levels, different main cooling system flowrates, and unequal reactor inlet temperatures from the two cooling loops. In addition, coolant temperature and flowrate distributions at the subassembly outlet measured in a zero power experiment are presented.

Maity et al. (2011) carried out thermal hydraulic studies to understand temperature dilution (defined as the difference between thermocouple reading and real temperature at the same location) experienced by core-temperature monitoring system of a sodium cooled fast reactor. The three-dimensional computational model was validated against experimental results of a water model. The analysis indicates the maximum possible dilution in fuel and blanket subassemblies to be $2.63 \mathrm{~K}$ and $46.84 \mathrm{~K}$, respectively. Shifting of thermocouple positions radially outward by $20 \mathrm{~mm}$ with respect to subassembly centers leads to an overall improvement in accuracy of thermocouple readings. It is also seen that subassembly blockage that leads to $7 \%$ flow reduction in fuel subassembly and $12 \%$ flow reduction in blanket subassembly can be detected effectively by the core-temperature monitoring system.

Di Piazza et al. (2014) carried out a CFD study on fluid flow and heat transfer in the Lead-cooled Fuel Pin Bundle of the ALFRED LFR DEMO. The authors developed a detailed thermo-fluid dynamic analysis at various levels of geometrical blockage. In particular, the closed hexagonal grid-spaced fuel assembly of the LFR ALFRED was modeled and computed. While the spacer grids were not included in the model, a conservative analysis has been carried out based on the current main geometrical and physical features. Results indicate that critical conditions, with clad temperatures $\approx 900^{\circ} \mathrm{C}$, are reached with geometrical blockage larger than $30 \%$ in terms of area fraction. The results show that two main effects can be recognized: a local effect in the wake/recirculation region downstream the blockage and a global effect due to the lower mass flow rate in the blocked subchannels. The former effect gives rise 
to a temperature peak behind the blockage and it is dominant for large blockages $(>20 \%)$, while the latter effect determines a temperature peak at the end of the active region and it is dominant for small blockages $(<10 \%)$. The blockage area was placed at the beginning of the active region, in such a way that both abovementioned phenomena can be seen. Different mass flow rates and different levels of geometrical blockage were imposed from preliminary system code simulations. Transient analyses with fully resolved SST-omega turbulence model were carried out and results indicate that a blockage of $\sim 15 \%$ (in terms of blocked area) leads to a maximum clad temperature around $800^{\circ} \mathrm{C}$, and this condition is reached in a characteristic time of 3-4 s without overshoot. Local clad temperatures around $1000^{\circ} \mathrm{C}$ can be reached for blockages of $30 \%$ or more. CFD simulations indicate that blockages (in terms of blocked area) greater than $15 \%$ could be detected by placing thermocouples in properly selected locations in the plenum region of the FA.

Kirsch (1975) presented experimental and theoretical investigations of the flow and temperature distribution in local recirculating flows in rod bundles, downstream of a blockage. A mean coolant temperature in this recirculation zone can be calculated from the dimension of the recirculation zone and the mass exchange rate with the main flow. Similarity analysis for recirculating flow in a simple geometry without rods shows that with a sufficiently high Reynolds number, similar geometry and similar heat distribution, the dimensionless temperature fields (in the bulk region) in recirculating flows are equal and independent of the Reynolds and Prandtl numbers. This result is also observed to be true for rod bundles, justifying temperature distribution measurements to be performed with water instead of sodium. Otherwise, the experimental study of temperature field on pins' surfaces due to a blockage, requires a liquid metal as coolant.

This work is focused on a CFD pre-test analysis of the new experimental test section called 'Blocked' Fuel Pin bundle Simulator (BFPS) that will be installed into the NACIE-UP (NAtural CIrculation Experiment-UPgrade) facility located at the ENEA Brasimone Research Center (Italy). RANS simulations were carried out adopting the ANSYS CFX commercial code. The loci of the peak temperatures and their width as predicted by the CFD simulations are used for determining the correct location of the pin bundle instrumentation.

\section{The NACIE-UP facility}

The results of the flow blockage simulations presented in this paper will be used to select the location of the thermocouples and other probes in the experiments carried out by a proper BFPS test section installed in the NACIE-UP facility.

NACIE-UP is a rectangular loop, which allows to perform experimental campaigns in the field of the thermal-hydraulics, fluiddynamics, chemistry control, corrosion protection and heat transfer and to obtain correlations essential for the design of nuclear power plant cooled by heavy liquid metals. It basically consists of two vertical pipes of O.D. 2.5 in. $(63.5 \mathrm{~mm})$ ), working as riser and down comer, connected by two horizontal pipes of O.D. $2.5 \mathrm{in} .(63.5 \mathrm{~mm})$ ). The whole height of the facility is about $7.7 \mathrm{~m}$, while the horizontal length is about $2.4 \mathrm{~m}$. A section of the facility is devoted to place the prototypical fuel pin bundle simulator (FPS) test sections in the lower part of the riser. In the last experimental campaign, performed in 2015, a wire-wrapped 19-pin $250 \mathrm{~kW}$ FPS was mounted. The test section for the blockage experiment will be placed in place of the previous one. A proper heat exchanger is placed in the upper part of the down comer.

NACIE-UP is made of stainless steel (AISI 304) and can use both lead and the eutectic alloy LBE as working fluid (about $2000 \mathrm{~kg}$, 2001 of total capacity). It was designed to work up to $550^{\circ} \mathrm{C}$ and $10 \mathrm{bar}$. The difference in height between the center of the heating section and the center of the heat exchanger is about $5.5 \mathrm{~m}$ and it is very important for the natural circulation. In the riser, an argon gas injection device ensures a driving force to sustain forced convection in the loop.

A schematic layout of the primary circuit is reported in Figure 1. The facility includes:

- The Primary side filled with LBE, with $2.5 \mathrm{in}$. $(63.5 \mathrm{~mm})$ pipes. It consists of two vertical pipes, working as riser and down comer, two horizontal pipes and an expansion tank;

- A Fuel Pin Simulator (19-pins) $250 \mathrm{~kW}$ maximum power, placed in the bottom of the riser of the primary side (it will be replaced by the new BFPS test section);

- A shell and tube HX with two sections, operating at low power (5-50 kW) and high power $(50-250 \mathrm{~kW})$. It is placed in the higher part of the down comer;

- A prototypical thermal flow meter $(0-20 \mathrm{~kg} / \mathrm{s})$, located in the cold leg;

- 3 bubble tubes to measure the pressure drops across the main components and the pipes;

- Differential pressure transducers (1 mbar accuracy) for the test section;

- Several bulk thermocouples to monitor the temperature along the flow path in the loop;

- The secondary side, filled with water at 16 bar, connected to the HX, shell side. It includes a pump, a pre-heater, an air-cooler, by-pass and isolation valves, and a pressurizer with cover gas;

- An ancillary gas system, to ensure a proper cover gas in the expansion tank, and to provide gas-lift enhanced circulation;

- A LBE draining section, with 0.5 in. $(12.7 \mathrm{~mm})$ pipes, isolation valves and a storage tank. 
The ancillary gas system has the function to ensure the cover gas in the expansion tank and to manage the gas-lift system in the riser for enhanced circulation regime.

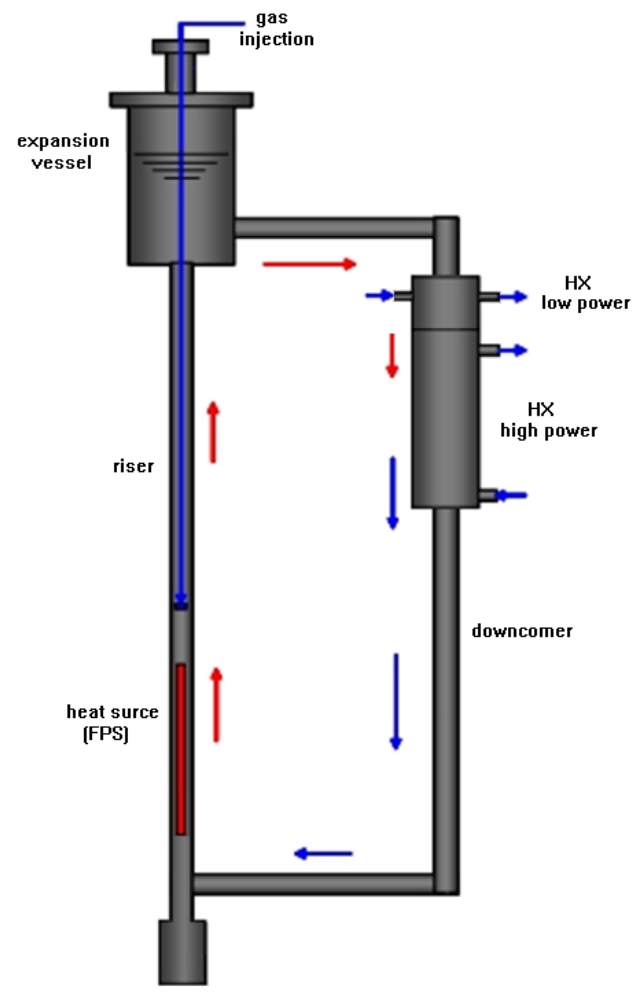

Figure 1 Technical drawing and main dimension of the NACIE-UP loop.

\section{The BFPS test section}

The Blocked Fuel Pin Simulator (BFPS) test section was designed in order to study the local and bulk effects of an internal blockage in a 19-pin LFR ALFRED DEMO-like FPS.

The heat source will consist of 19 electrical pins with an active length $L_{\text {active }}=600 \mathrm{~mm}\left(L_{\text {total }}=2000 \mathrm{~mm}\right.$, including the non-active length) and a diameter $D=10 \mathrm{~mm}$. The pitch to diameter ratio is $P / D=1.4$. The maximum external pin heat flux will be $\approx 0.7 \mathrm{MW} / \mathrm{m}^{2}$. The pins will be placed on a hexagonal layout by a suitable wrapper, while two grids will maintain the pin bundle in the correct position. The total power of the pin fuel bundle is $\approx 250 \mathrm{~kW}$.

This fuel pin bundle configuration is relevant for the ALFRED's core thermal-hydraulic design. ALFRED is a flexible fast spectrum research reactor $\left(300 \mathrm{MW}_{\text {th }}\right.$ ). The main parameters of BFPS test section and ALFRED core are reported in Table 1 for comparison.

\begin{tabular}{|c|c|c|}
\hline Parameter & BFPS & ALFRED FA \\
\hline$D[\mathrm{~mm}]$ & 10 & 10.5 \\
\hline$P / D$ & 1.4 & 1.32 \\
\hline$Q[\mathrm{~kW}]$ & 250 & - \\
\hline$Q_{\text {pin }}[\mathrm{kW}]$ & 13 & - \\
\hline$q^{\prime \prime}\left[\mathrm{MW} / \mathrm{m}^{2}\right]$ & 0.7 & $0.7-1$ \\
\hline$v[\mathrm{~m} / \mathrm{s}]$ & 0.8 & 1.1 \\
\hline
\end{tabular}




\begin{tabular}{|c|c|c|}
\hline$N_{\text {pins }}$ & 19 & 127 \\
\hline$L_{\text {active }}[\mathrm{mm}]$ & 600 & 600 \\
\hline$L_{\text {plenum }}[\mathrm{mm}]$ & 500 & 500 \\
\hline
\end{tabular}

\section{Table 1 Comparison of the main parameters between the BFPS test section and ALFRED core}

The goals of the experimental campaigns planned on the NACIE-UP loop facility with the BFPS bundle are:

- measurement of the pin wall temperature both with and without blockage by embedded thermocouples;

- measurement of the subchannel temperature;

- Heat Transfer Coefficient (HTC) evaluation;

- axial temperature profiles in the wrapper and in the subchannels;

- check the presence of hot spots and localized peak of temperature;

- evaluation of the thermal mixing above the pin bundle;

A preliminary drawing of the BFPS test section is shown in Figure 2. A spacer grid is located at the beginning of the active region where the coupling flange is present. Without blockage, the test section allows to characterize the flow and the heat transfer in the FA. By opening the coupling flange located upstream the active region and by closing grid subchannels by caps, proper blockages at the beginning of the active region can be achieved.

The central spacer grid will be the key component of the flow blockage experimental campaign and it is specifically described in the following.

The central grid in its unblocked configuration is reported (viewed from the top) in Figure 3, with inlet pipe and pin numeration for reference. The grid has an external circular shape and two notches for the correct position of the component between the central flanges. The inner hexagonal key of the grid has the same dimension of the bundle one $(\mathrm{H}=62 \mathrm{~mm})$. The 19 circular holes have the same dimension and pitch of the bundle while the septa among them and the grid will have the lowest thickness achievable without compromise the structural integrity of the component and the right installation of the caps; anyway, the septa location is fixed.

For the flow blockage configuration, several caps will be displaced on the different holes of the central grid; those caps will be small thin plates of appropriate shape positioned by moving rods from the bottom to fix a configuration.

Pins and LBE temperature downstream the blockage will increase due to the lower cooling rate in this region. The extension and magnitude of those hot regions are strictly related to the blockage type and blocked flow area of the grid.

The test section will be instrumented with 100 thermocouples. The location of the thermocouples in the system is fixed according to the results of the present pre-test analysis. 


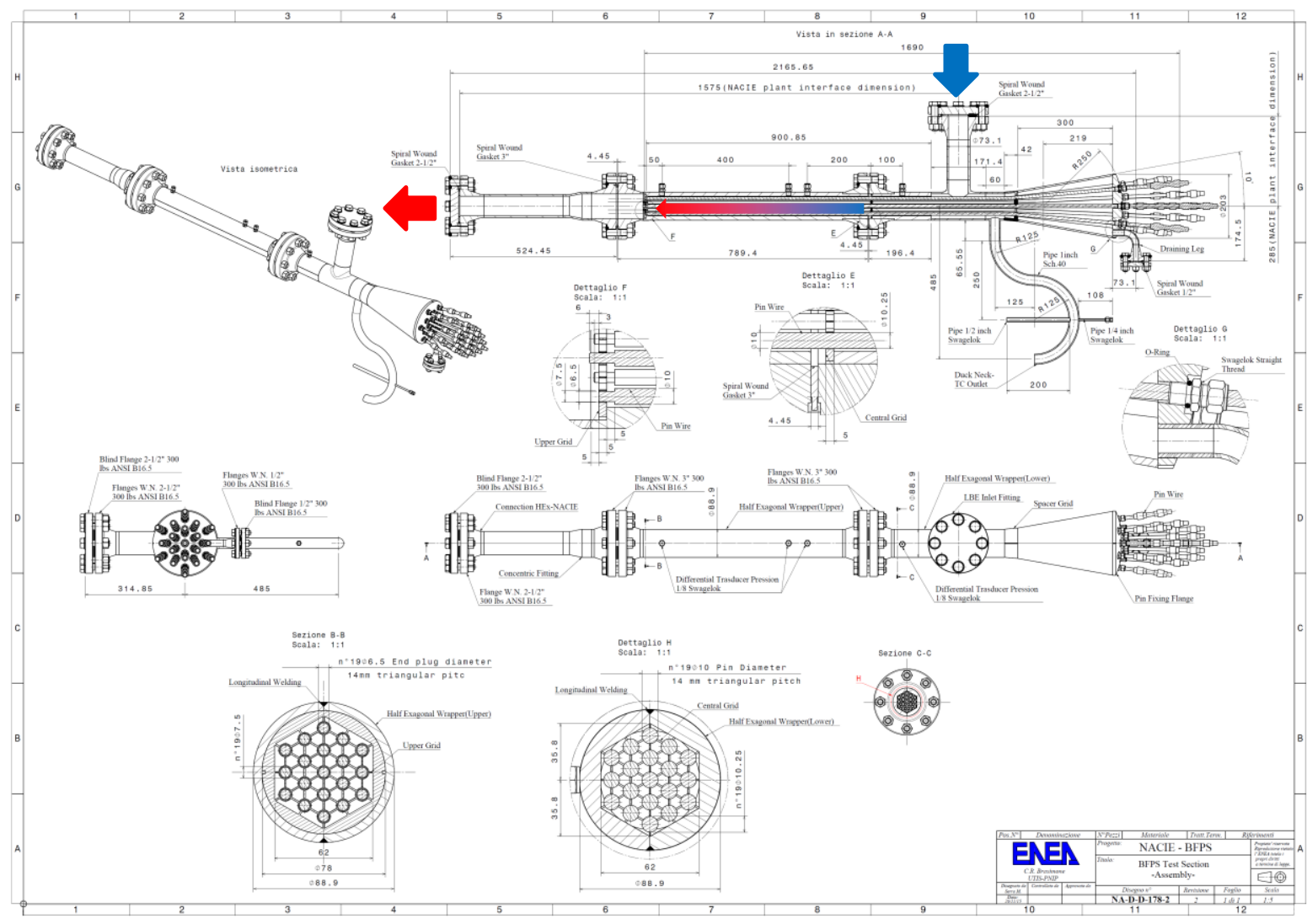

Figure 2 Preliminary mechanical drawing of the BFPS test section.

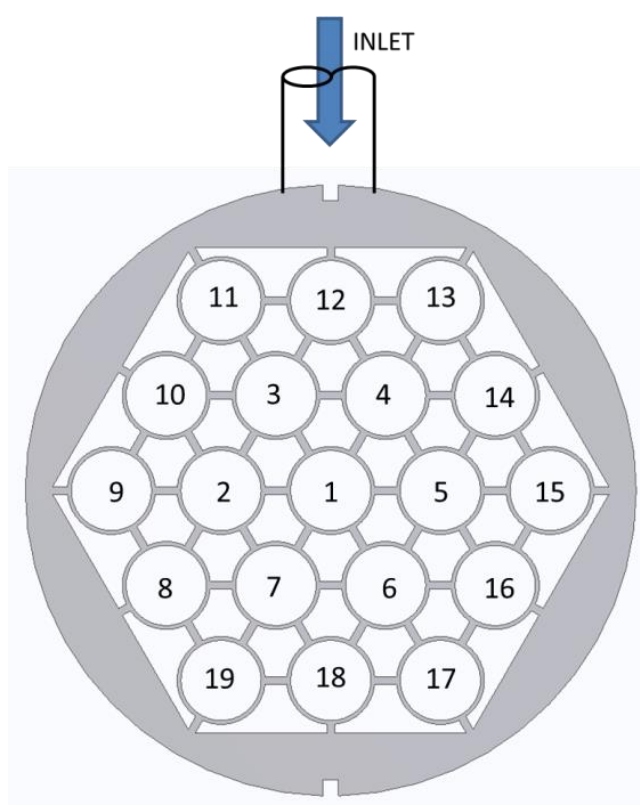

Figure 3 Sketch of the central grid in unblocked configuration (viewed from the top) 


\section{Numerical models and methods}

For the CFD pre-test analysis of the experiment, the BFPS test section was modeled. A sketch of the computational domain is shown in Figure 4 which includes (from bottom to top) the developing region, the active region ("Pins"), and the mixing region downstream of the FA. The inlet bend is not included in these simulations because its position is not fixed yet. Anyway, further sensitivity analyzes including the inlet bend, have shown that the previous simulations (without bend) were conservative (predicting higher temperature behind the blockage) but anyway hot-points locations and critical regions for instrumentation are the same. The conjugate heat transfer in the hexagonal wrapper and in the coupling flange was also accounted. The importance of the conjugate heat transfer in this kind of test sections was assessed in previous works; see Di Piazza (2015) and Doolaard (2015). The meshes developed are fully unstructured both in the fluid and solid domains. The number of nodes is inflated near the solid walls for the resolution of the viscous sub-layer $\left(y^{+}=1\right.$ for the highest Re case $)$ and at least 4 nodes in the viscous sub-layer.

The general-purpose code ANSYS CFX 15 was used for all the numerical simulations presented in this paper. The code employs a coupled technique, which simultaneously solves all the transport equations in the whole domain through a false time-step algorithm. The linearized system of equations is preconditioned in order to reduce all the eigenvalues to the same order of magnitude.

Constant mass flow rate, temperature at inlet and pressure at outlet were applied as boundary conditions for the simulations. The external surface of the hexagonal wrapper is considered adiabatic, being insulated with $100 \mathrm{~mm}$ of thermal insulator.

The SST (Shear Stress Transport) k- $\omega$ model by Menter (1994) was extensively used in this paper. It is formulated to solve the viscous sub-layer explicitly, and requires several computational grid points inside the viscous sub-layer. The turbulent Prandtl number was set to 1.5, according to the literature suggestions (Cheng and Tak, 2006). Buoyancy forces were neglected (conservative approach).

Four meshes have been developed and tested to perform a mesh independence analysis, with 10, 15, 19, 24 million nodes on the whole domain and $y^{+}=1$ for all the computational grids (see Table 2). Because the results for fluid flow, heat transfer and temperature (maximum and at outlet) of the unblocked case were comparable to a very high degree among the 4 meshes, the mesh $\mathrm{C}$ with 19 million nodes was selected representing a good compromise between computational requirements and numerical accuracy. Two views of mesh $\mathrm{C}$ are shown in Figure 5.

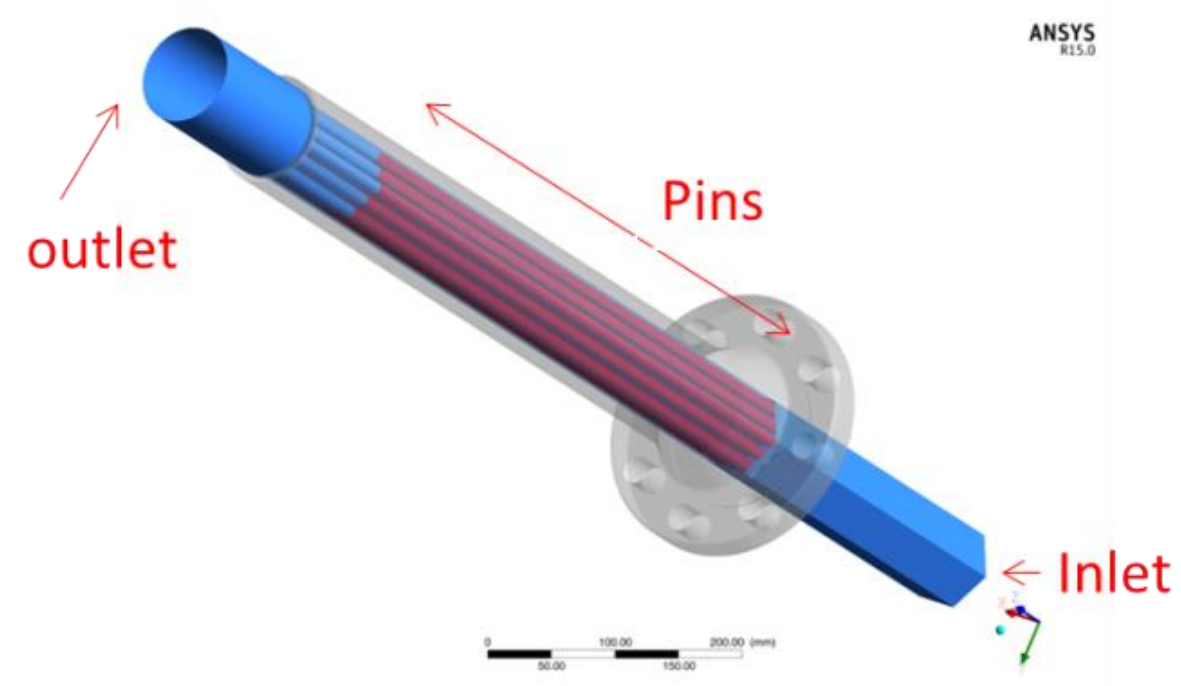

Figure 4 Sketch of the computational domain.

\begin{tabular}{|c|c|c|c|c|}
\hline Mesh & $\begin{array}{c}\text { Million nodes } \\
{[-]}\end{array}$ & $\begin{array}{c}\text { f Darcy } \\
{[-]}\end{array}$ & $\begin{array}{c}\text { Nu } \\
{[-]}\end{array}$ & $\mathbf{T}_{\text {pin, max }}\left[{ }^{\circ} \mathbf{C}\right]$ \\
\hline A & 10 & 0.01182 & 23.86 & 267.6 \\
\hline B & 15 & 0.01144 & 17.31 & 269.8 \\
\hline C & 19 & 0.01130 & 16.48 & 272.4 \\
\hline D & 24 & 0.01131 & 16.52 & 272.6 \\
\hline
\end{tabular}

Table 2 Mesh adopted for the grid-independence study, with the main overall output parameters. 

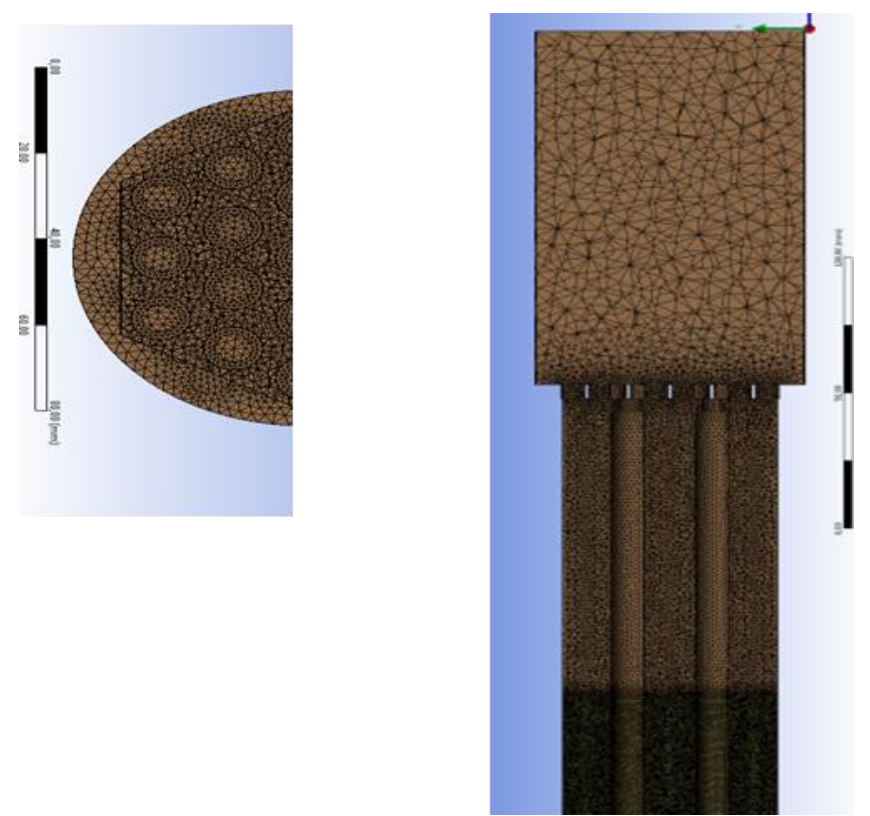

Figure 5 Computational mesh $\mathrm{C}$ adopted for the simulations

Four different blockage types were chosen for the pre-test analysis. These are shown in Figure 6 where type 0 refers to the unblocked case. The mechanical method for implementing the blockage (i.e. how to block the flow in the subchannels) is via a stainless steel cap fixed to the grid spacer with push\&pull rods, properly fitted according to the type and location of the blockage. A pre-test matrix was prepared for defining the different blockage types at different mass flow rates. In the test cases presented here, the mass flow rate was kept constant at $16 \mathrm{~kg} / \mathrm{s}$, corresponding to $108 \mathrm{~kg} / \mathrm{s}$ in the ALFRED FA (75\% of the nominal flow rate), and it is the maximum flow rate achievable in the NACIE-UP facility (maximum axial extension of the wake region behind the blockage). The power of the FPS was $94.2 \mathrm{~kW}$ to have an inlet-outlet temperature increase of $40^{\circ} \mathrm{C}$ (for BFPS safe operation). The inlet temperature is $200^{\circ} \mathrm{C}$, to protect test section from damages during the experiments.

Only the most relevant cases of the complete computational test matrix are presented here, and are summarized in Table 3.

\begin{tabular}{|c|c|c|c|c|c|}
\hline Case & Blockage type & Mass flow rate [kg/s] & Re $_{\text {BFPS [-] }}$ & Power [kW] & Inlet temperature [ ${ }^{\circ} \mathbf{C}$ ] \\
\hline 0 & 0 & 16 & 46663 & 94.2 & 200 \\
\hline 1 & 1 & 16 & 46663 & 94.2 & 200 \\
\hline 2 & 2 & 16 & 46663 & 94.2 & 200 \\
\hline 3 & 3 & 16 & 46663 & 94.2 & 200 \\
\hline 4 & 4 & 16 & 46663 & 94.2 & 200 \\
\hline 5 & 5 & 16 & 46663 & 94.2 & 200 \\
\hline
\end{tabular}

Table 3 Test matrix presented in the paper.

The mass flow rate was kept constant at the different degree of blockage. This is justified by the fact that in the real ALFRED FA geometry, blockage degrees up to $30 \%$ would not affect significantly the flow rate (Di Piazza, 2014) and the interest is focused on the local phenomena.

Figure 7 shows the clad temperature distribution in the active region for the unblocked case 0 . The temperature gradient is basically axial as expected with a maximum temperature of the cladding is $261^{\circ} \mathrm{C}$, with hot points in the corner regions. Figure 8 (left) shows the clad temperature distribution in the region behind the blockage for blockage type 1 (sector blockage, case 1), while Figure 8 (right) shows the LBE temperature in the stagnation region immediately after the blockage. A local temperature peak (Figure 8 right) in the region $100 \mathrm{~mm}$ behind the blockage is clearly visible with a maximum temperature of about $360^{\circ} \mathrm{C}$, well above the maximum temperature of the unblocked case. The local temperature increase due to the blockage is about $150^{\circ} \mathrm{C}$. As evidenced in other computational and theoretical studies (Di Piazza, 2015) (Kirsch, 1975) (Roelofs, 2012), the reason for the local temperature peak is the vortex generated downstream by the obstacle represented by the blockage; in the central, stagnation point of the vortex heat is 
exchanged only by conduction and the temperature increases. This hydrodynamic effect is shown in Figure 9, where temperature field and secondary flow field (left) are shown at the cross-sections at $z=10,20,30,45 \mathrm{~mm}$ from the blockage (located at $z=0 \mathrm{~mm}$ at the beginning of the active region, first grid) for case 1 (sector blockage). A vortex due to the blockage obstacle is clearly evidenced between $z=10 \mathrm{~mm}$ and $45 \mathrm{~mm}$ with a maximum of the secondary flow (see Figure 9 (center) at $\mathrm{z}=45 \mathrm{~mm}$ ). The temperature field shows a peak value in planes $z=20,30 \mathrm{~mm}$ in the stagnation region.

Figure 10 shows the temperature distribution for all the cases simulated at $16 \mathrm{~kg} / \mathrm{s}$ at the outlet section in the mixing region; please note that the color scales adopted are different. The trace of the blockage is clearly visible in all cases. Figure 12 shows the temperature distribution in a radial line at the outlet section. For the unblocked case (Case 0), mixing guarantees a maximum spatial difference of temperature of about $1.5^{\circ} \mathrm{C}$, while in the blocked cases the spatial temperature distribution clearly shows the location of the underlying blockage with a temperature difference of $15{ }^{\circ} \mathrm{C}$ for Case 1 and $28{ }^{\circ} \mathrm{C}$ for Case 5 . The relevant difference both in the value of the temperature in a specific location as well as in the spatial distribution of the temperature confirms the possibility to detect the internal blockage at this level of blockage by putting thermocouples in properly selected locations of the mixing region of the BFPS test section (Naveen Raj, 2016).

The experimental investigation of the temperature gradients in the mixing region of the test section is crucial to confirm this possibility.

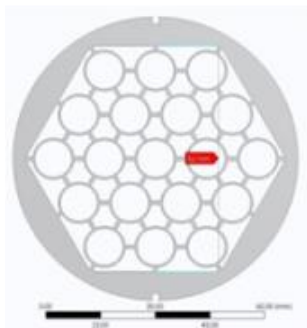

0

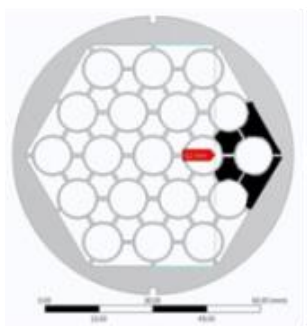

3

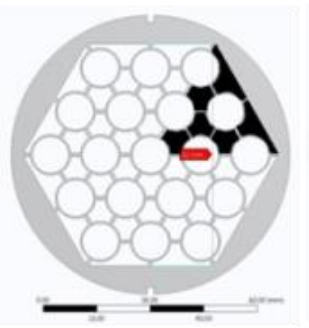

1

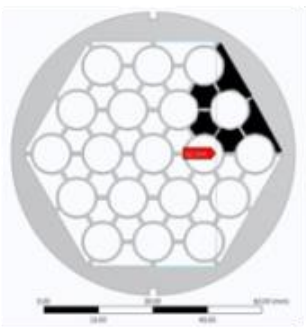

4
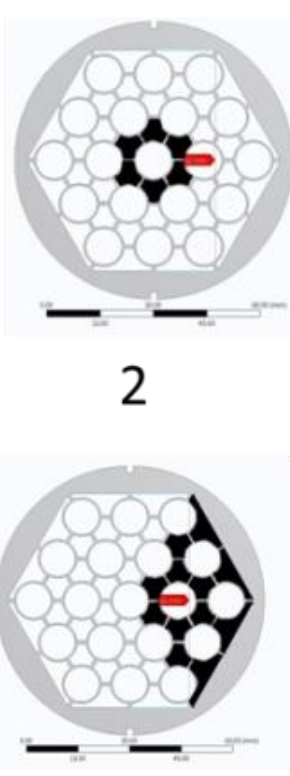

5

Figure 6 Different blockage types: (0) unblocked; (1) sector; (2) central; (3) corner; (4) edge; (5) two sectors. 


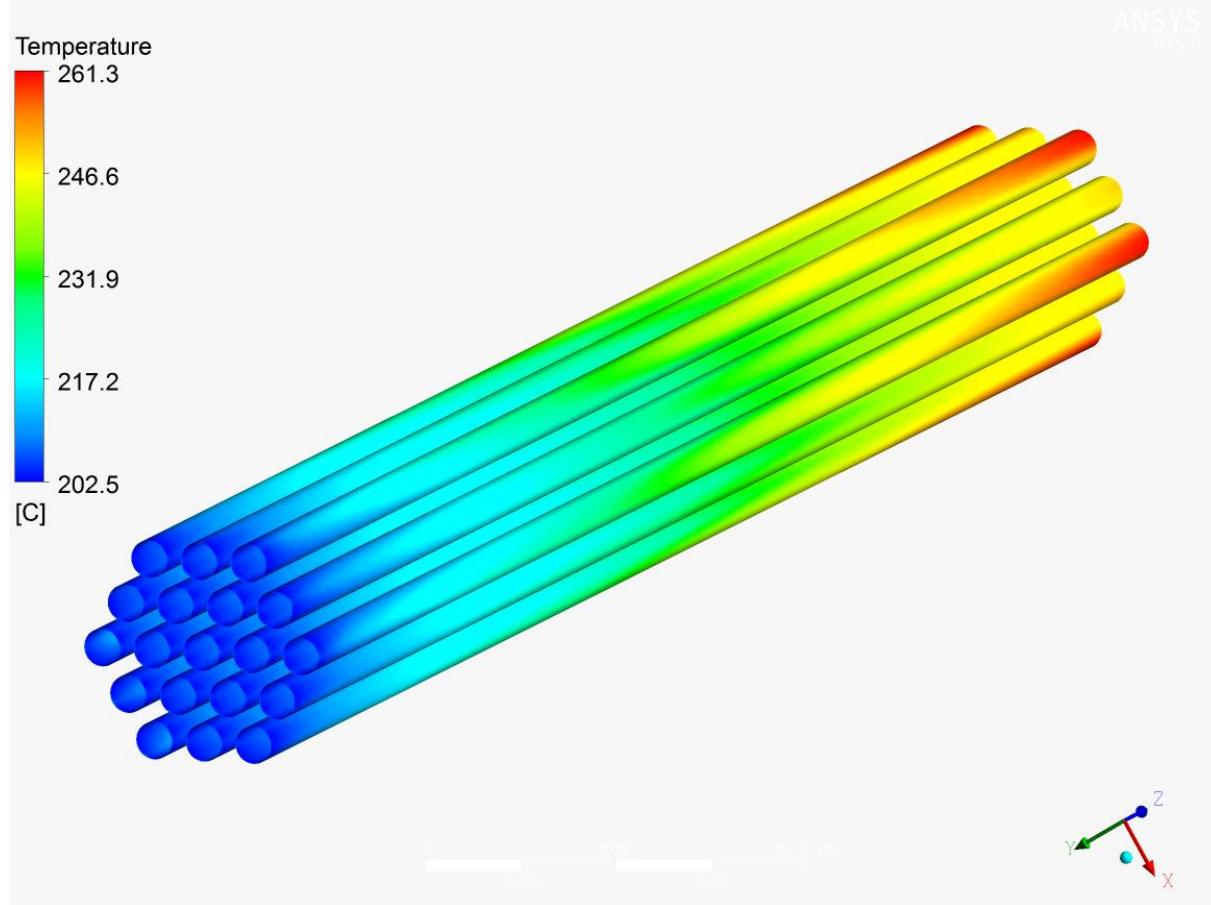

Figure 7 Pin clad temperature field in the unblocked case.
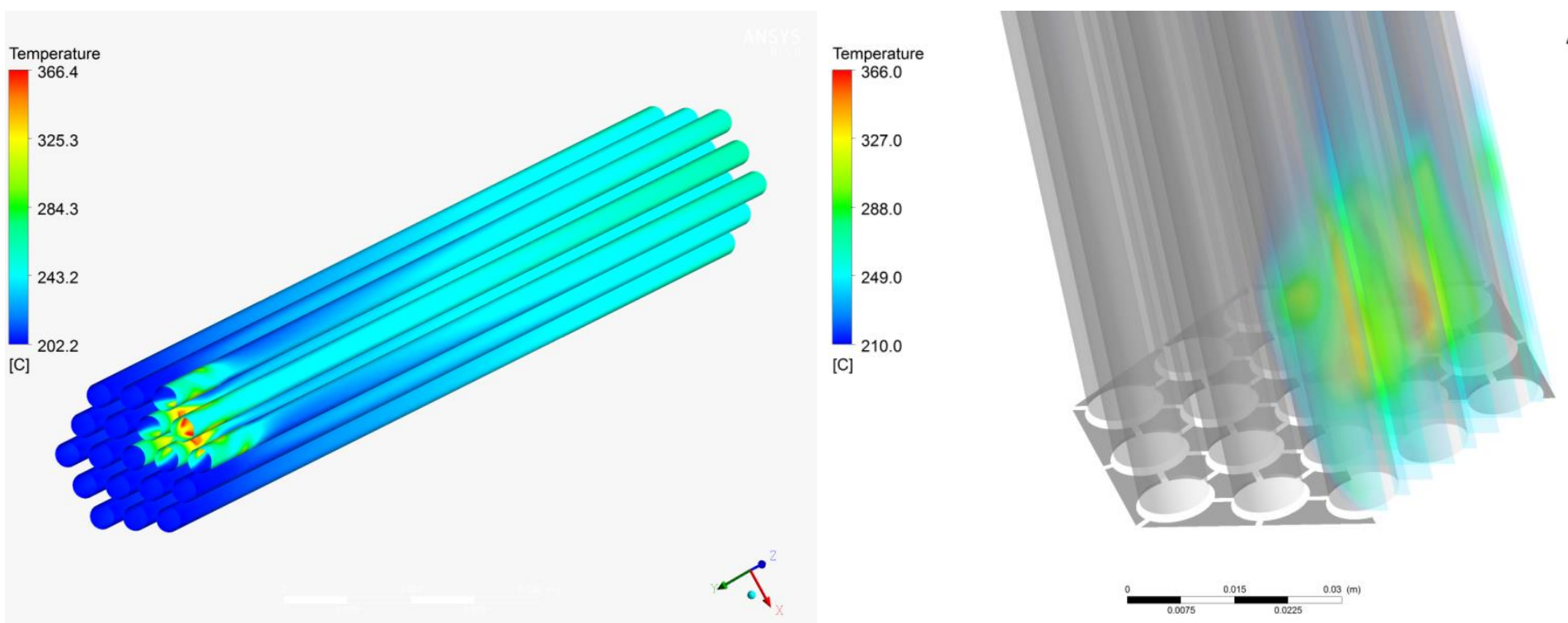

Figure 8 Clad temperature field in the region behind the blockage (Case 1) on the left and LBE temperature in the stagnation region on the right. 


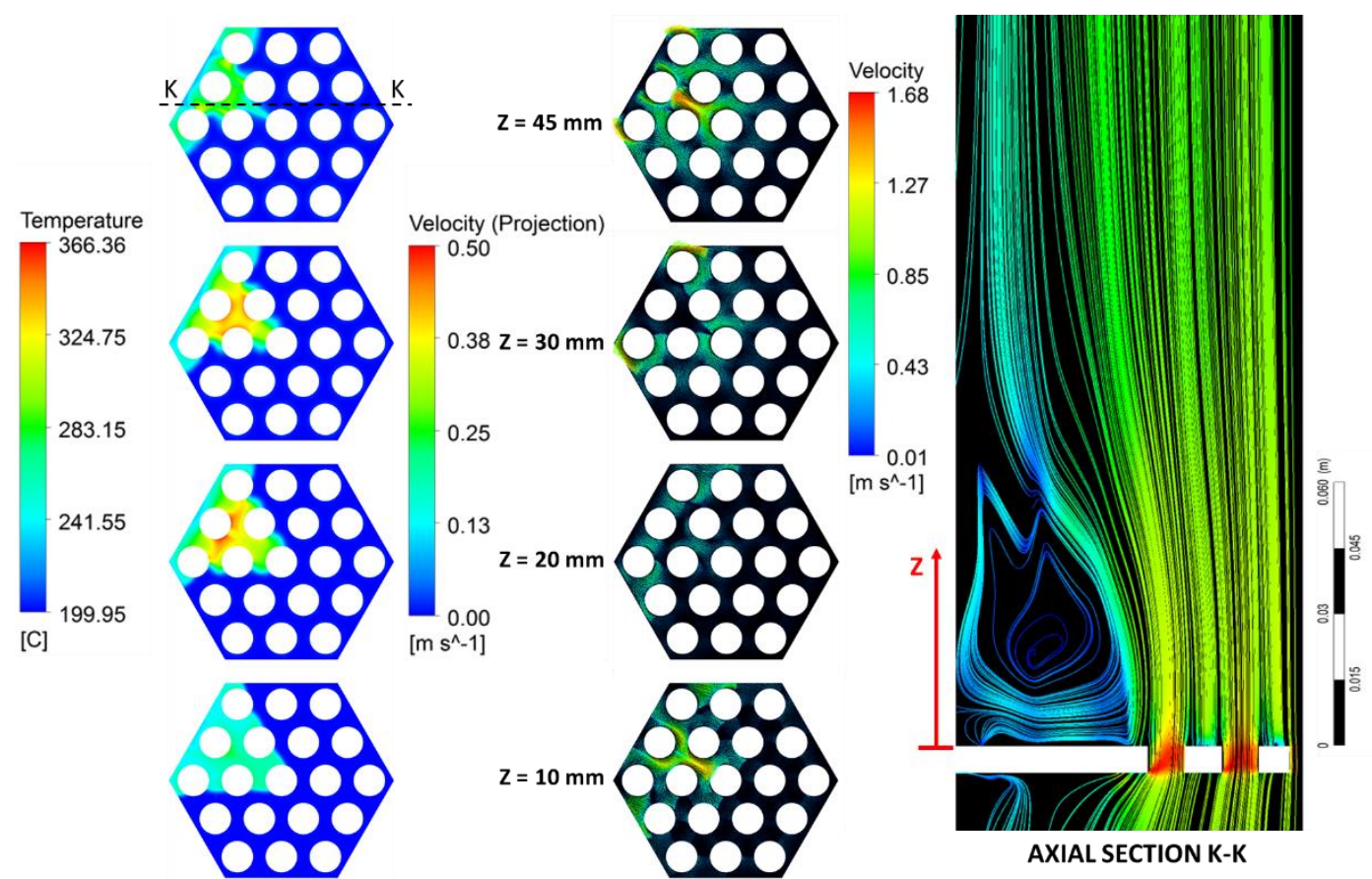

Figure 9 Temperature field and secondary vector plot (left) on four different planes located at $\mathrm{z}=10,20,30,40,45 \mathrm{~mm}$ from the beginning of the active region. Recirculation region over the sector blockage (right). 

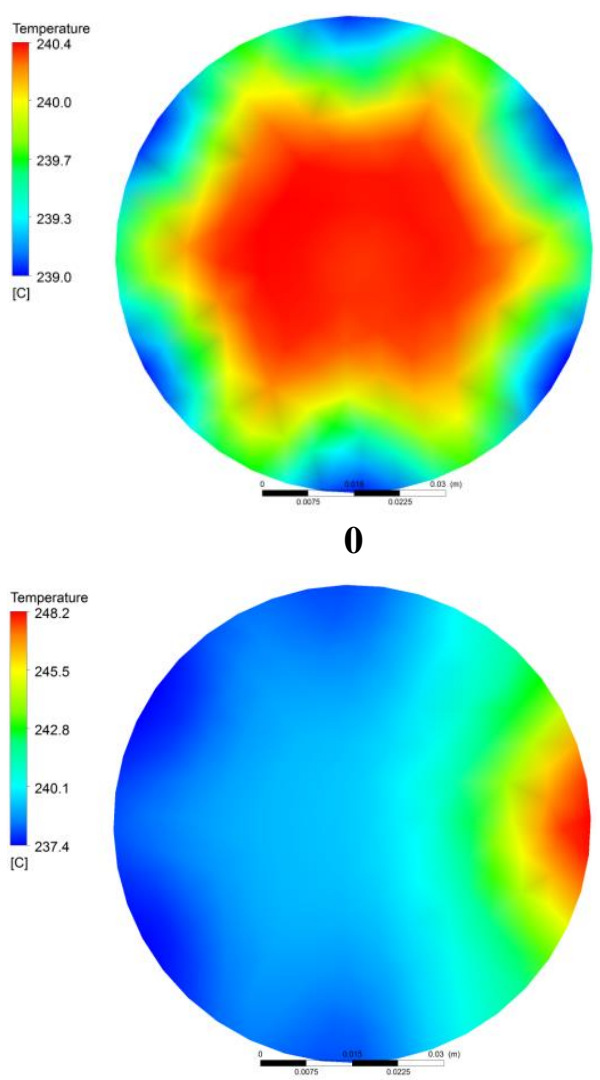

3
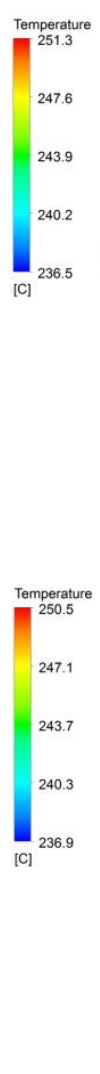

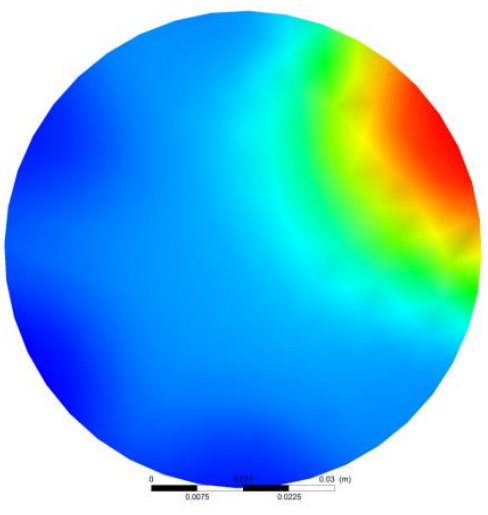

1

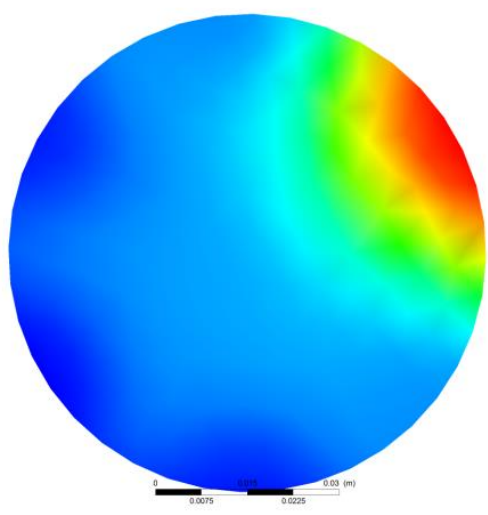

4
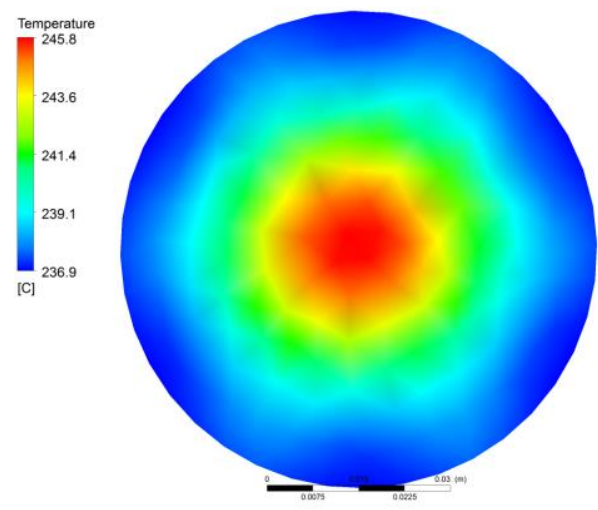

2
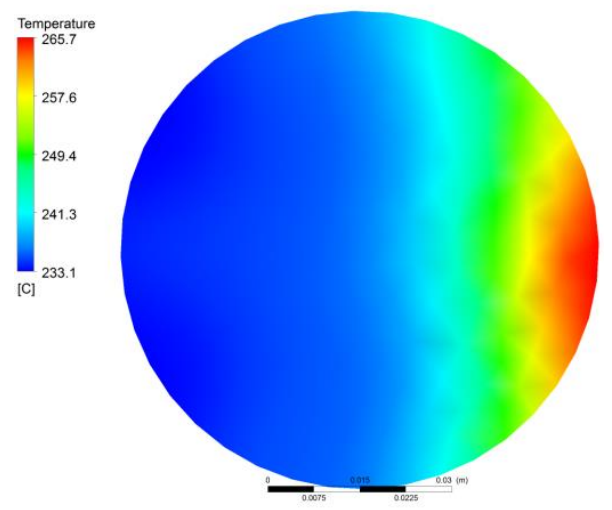

5

Figure 10 Temperature distribution on the outlet section of the mixing region $(16 \mathrm{~kg} / \mathrm{s})$ for case 0 (unblocked), 1 (sector blockage), 2 (central blockage), 3 (corner blockage), 4 (edge blockage), 5 (two sector blockage).
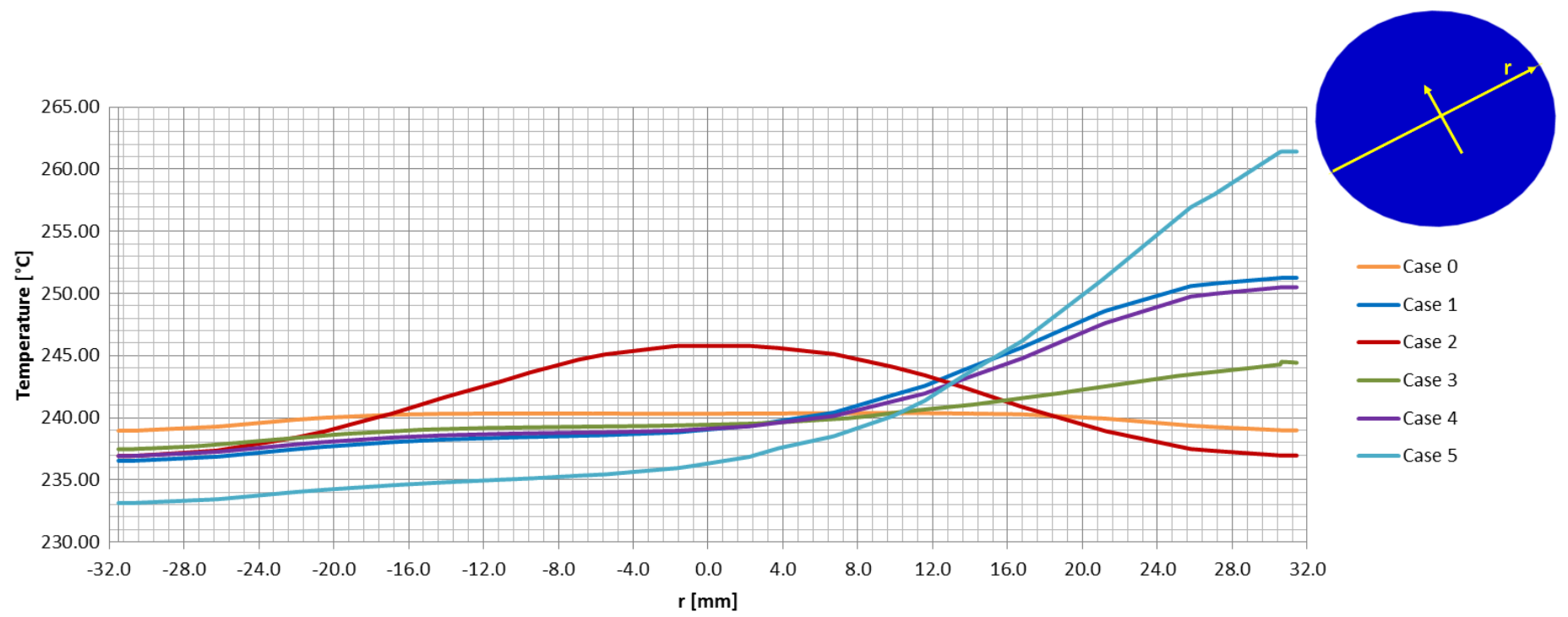

Figure 11 Temperature profile for all the cases simulated on a line placed at the outlet section of the model (top right sketch)

Figure 12 shows the maximum temperature profiles along the stream wise axial direction for all simulated cases, with the axial distance starting from the beginning of the active region. Most of the cases have a similar maximum temperature behind the blockage 
around $360^{\circ} \mathrm{C}$, about $150^{\circ} \mathrm{C}$ difference with respect to the unblocked case. The region of influence behind the blockage is limited to 50-100 $\mathrm{mm}$ in most cases, and $200 \mathrm{~mm}$ for the larger two-sector blockage (case 5). This result is in line with previous studies (Di Piazza, 2015) (Kirsch, 1975) and the region of influence is tied to the extension of the blocked area. Typically, an elongated recirculation vortex of aspect ratio 2-3 behind the blockage is present. A larger blockage will lead to a longer vortex and to a larger region of influence.

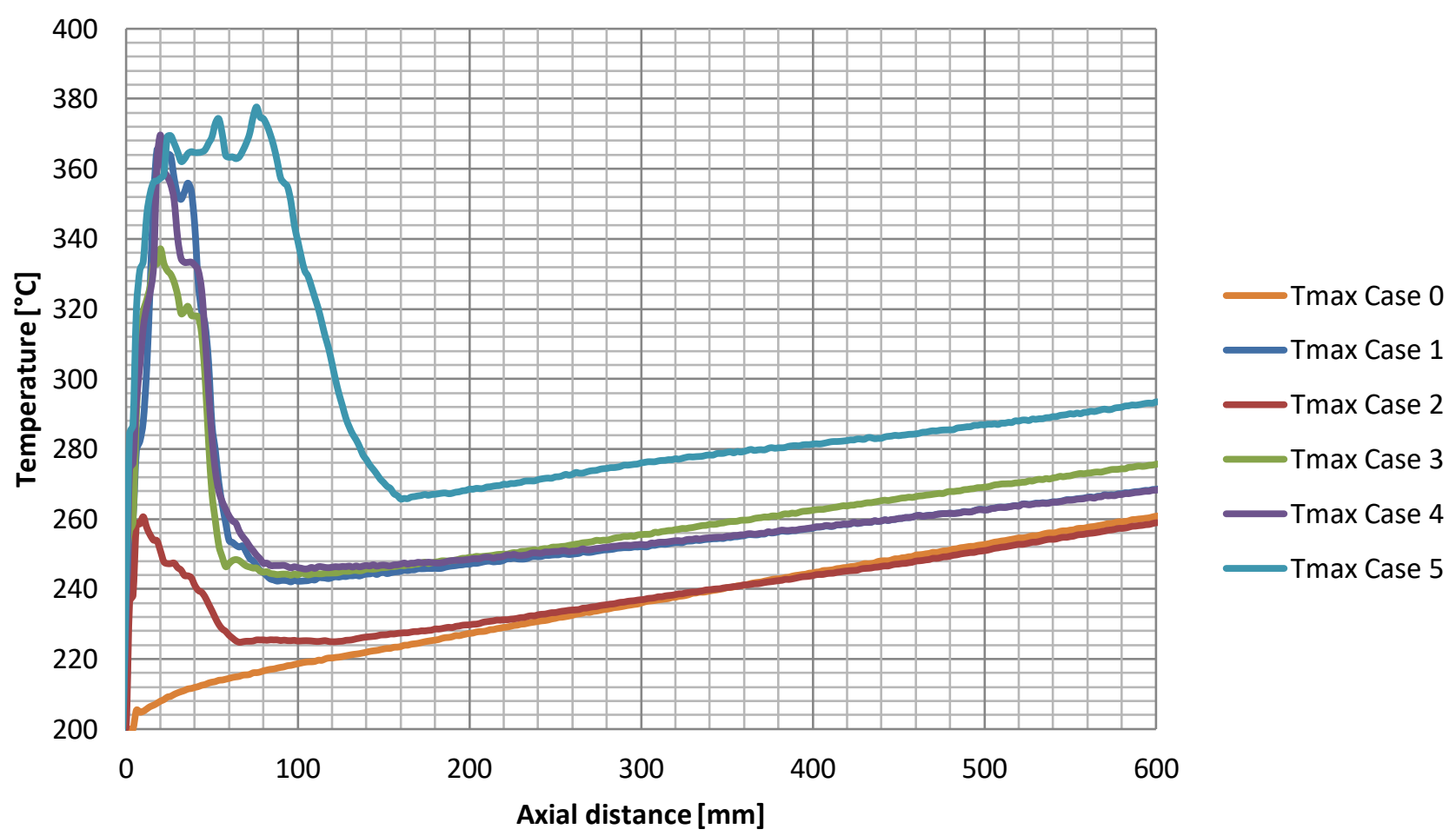

Figure 12 Maximum temperature profile along the stream-wise axial direction for the 6 cases investigated.

The previous numerical simulations with different blockage types were also performed at lower mass flow rates $(4 \mathrm{~kg} / \mathrm{s}$ and $8 \mathrm{~kg} / \mathrm{s})$ keeping the same inlet-outlet bulk temperature increase $\left(40^{\circ} \mathrm{C}\right)$; their main input data are reported in Table 4 .

\begin{tabular}{|c|c|c|c|c|c|}
\hline Case & Blockage type & Mass flow rate [kg/s] & Re $_{\text {BFPs }[-]}$ & Power [kW] & Inlet temperature $\left[{ }^{\circ} \mathbf{C}\right.$ ] \\
\hline $0-8$ & 0 & 8 & 23332 & 47.1 & 200 \\
\hline $1-8$ & 1 & 8 & 23332 & 47.1 & 200 \\
\hline $2-8$ & 2 & 8 & 23332 & 47.1 & 200 \\
\hline $3-8$ & 3 & 8 & 23332 & 47.1 & 200 \\
\hline $4-8$ & 4 & 8 & 23332 & 47.1 & 200 \\
\hline $5-8$ & 5 & 8 & 23332 & 47.1 & 200 \\
\hline $0-4$ & 0 & 4 & 11666 & 23.57 & 200 \\
\hline $1-4$ & 1 & 4 & 11666 & 23.57 & 200 \\
\hline $2-4$ & 2 & 4 & 11666 & 23.57 & 200 \\
\hline $3-4$ & 3 & 4 & 11666 & 23.57 & 200 \\
\hline $4-4$ & 4 & 4 & 11666 & 23.57 & 200 \\
\hline $5-4$ & 5 & 4 & 11666 & 23.57 & 200 \\
\hline
\end{tabular}

Table 4 Test matrix of the pre-test study 
The most interesting parameter to be compared among the different cases is the maximum temperature on the pins and the extent of the "hot region" keeping a constant blockage type. This is shown in the different graphs of Figure 13. The axial dimension of the recirculation region is not strongly dependent from mass flow rate but it is mainly tied to the blockage area and type; in particular its maximum width is achieved for blockage 5 (two sector blockage) and $16 \mathrm{~kg} / \mathrm{s}$ where it reaches $200 \mathrm{~mm}$ at least.

On the other hand, the peak cladding temperature increases with the blocked area and with the mass flow rate. This latter effect on the mass flow rate is due to the fact that the test matrix is designed with a constant inlet-outlet bulk temperature, and therefore the heat flux increases linearly with the mass flow rate. For example, for blockage 1, the power of the FPS is $23.57,47.1,94.2 \mathrm{~kW}$ for $4,8,16$ $\mathrm{kg} / \mathrm{s}$, respectively.

The separate influence of mass flow rate on the temperature peak for a fixed blockage configuration is shown in Figure 14 in the case of blockage 1 (sector) and a constant power of $47.1 \mathrm{~kW}$. The temperature peak increases when the mass flow rate decreases, while the extension of the hot region is nearly the same, as clearly expected from intuition.
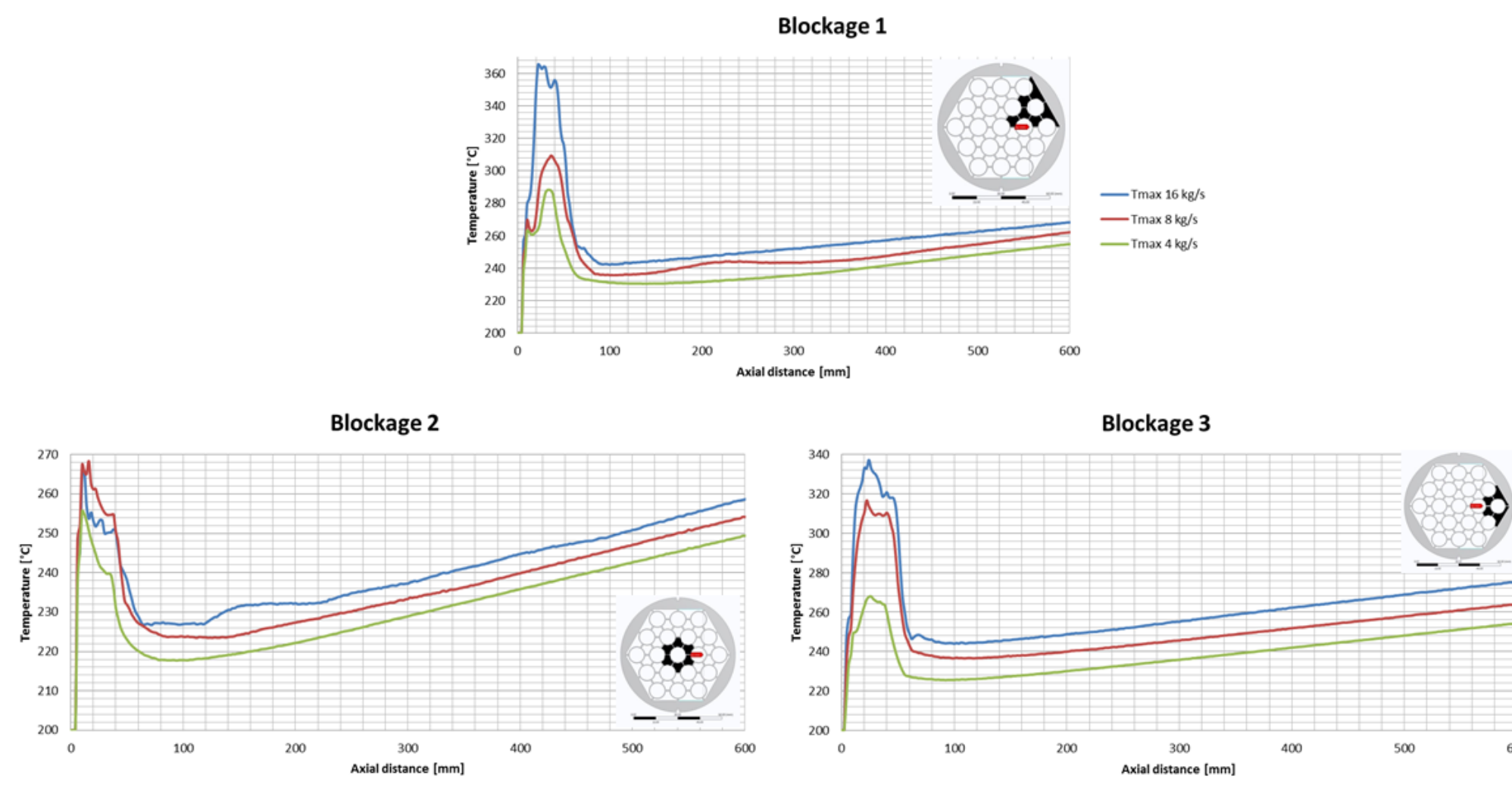

Blockage 4
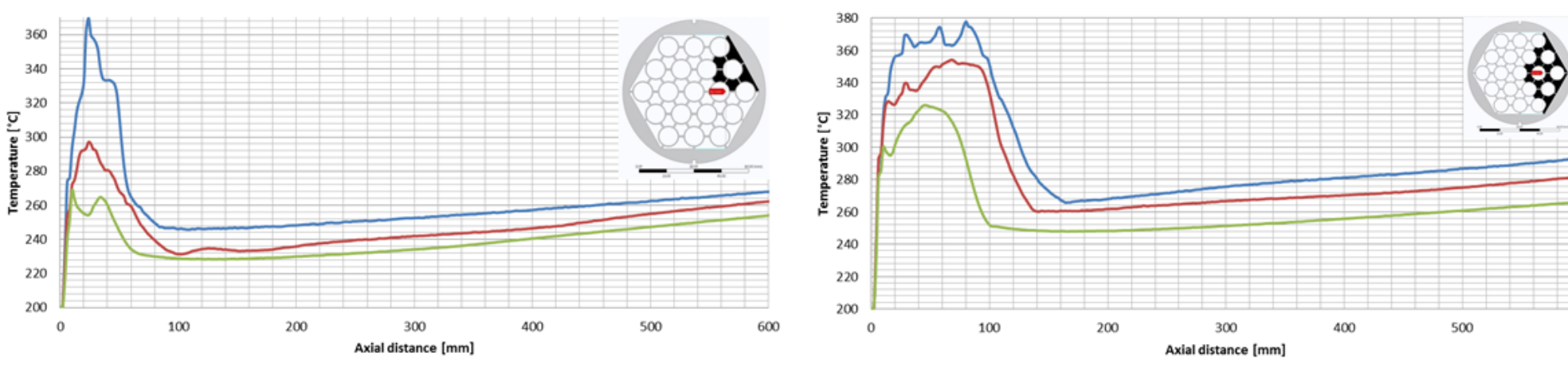

Figure 13 Comparison of maximum temperature and 'hot region' extension for different blockages and at different mass flow rates. 


\section{Blockage 1 -Constant power 47.1 kW}

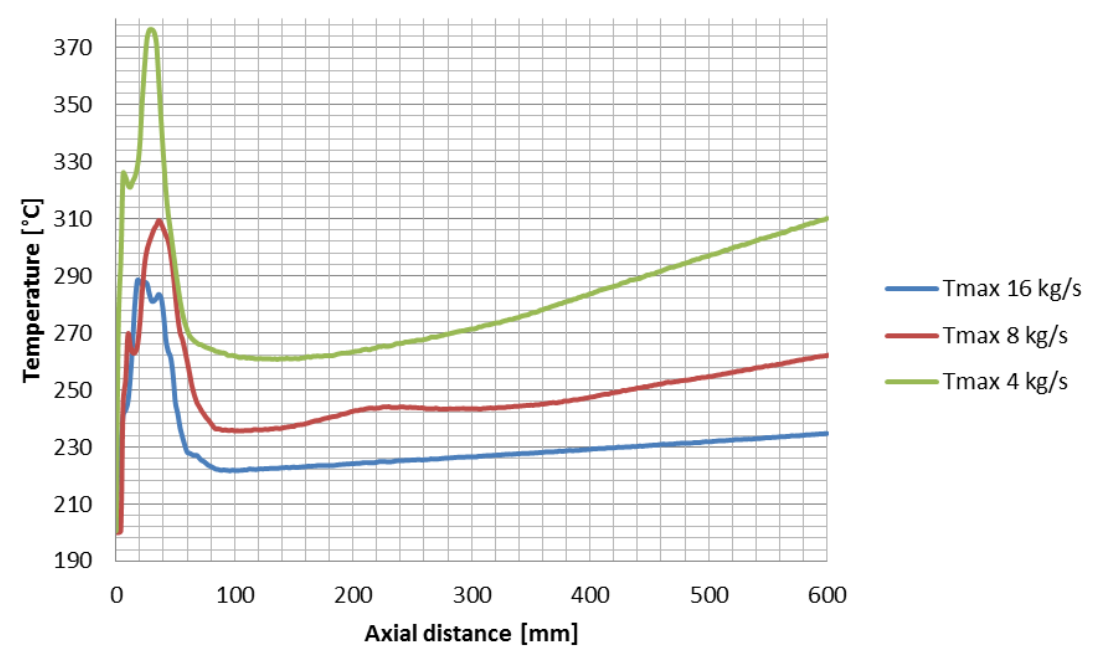

Figure 14 Maximum temperature along the axis at different mass flow rates and at a constant bundle power of $47.1 \mathrm{~kW}$.

The present results suggest how to position of the instrumentation in the test section. The blocked region will be monitored with 0.35 $\mathrm{mm}$ wall-embedded thermocouples, most of them located on the hottest pins in the region of influence. The remaining thermocouples are positioned in order to measure bulk and wall temperatures in the different subchannels and to characterize heat transfer in different conditions. The generic section of the FPS was divided in 36 subchannels, see Figure 15, corresponding to the grid passages at the beginning of the active region, see Figure 3 for comparison. The bundle was divided in 6 sectors from A to F. The code identifying the subchannel, e.g. B2, refers to the sector (e.g. letter 'B') and to the rank (e.g. number '2').

The thermocouple locations are shown in Figure 16, with the instrumented pins colored in red. Pins 1, 2, 5, 15 will be equipped with wall embedded thermocouples on a generatrix parallel to the pin axis, and sub-channel B2 will be instrumented with $0.5 \mathrm{~mm}$ bulk thermocouples.

Sixteen different levels will be considered for the four generatrices and the sub-channel: $z=10,20,30,40,50,60,70,80,90,100,150$, $200,300,400,500,600 \mathrm{~mm}$ starting from the beginning of the active region of the pins. Plane at $z=550 \mathrm{~mm}$ will be instrumented as in the following to characterize the heat transfer in the unblocked case:

- $\quad$ ins $1,2,4,5,7,9,14,15$ will be instrumented with wall embedded thermocouples;

- sub-channels B1, B2, B5, E1, E5 and the corner sub-channels across B5/C4 and E5/F4 will be instrumented with bulk thermocouples $0.5 \mathrm{~mm}$ thickness placed at the center of the subchannel;

- An additional bulk thermocouple of $0.35 \mathrm{~mm}$ thickness is placed in subchannel B1 at a distance of $1 \mathrm{~mm}$ from the wall.

This instrumentation will allow to collect data on temperature distribution in the case of blockage and to characterize the heat transfer in the unblocked condition by measuring cold spots in the side subchannels and the heat transfer coefficients. This will be a fundamental characterization of the ALFRED FA for the different ranks of subchannels.

Additional instrumentation (24 TCs) will be placed in the $500 \mathrm{~mm}$ mixing region of the test section to collect data for CFD code validation and HLM thermal mixing. TCs will be placed downstream the FPS at different axial, radial and azimuthal positions.

This choice is justified by the temperature trend at the outlet section of the CFD model for the different blockage types and different mass flow rates simulated and reported in Figure 11, Figure 17 and Figure 18 for the 16, 8 and $4 \mathrm{~kg} / \mathrm{s} \mathrm{cases}$ respectively. The radial temperature distribution in the mixing region over the pin bundle, in particular its shape and temperature maxima, could be adopted as a track for detecting the faulted FAs (affected by blocked sub-channels). 
Figure 15 Sketch of a section of the new FPS for the NACIE-UP facility viewed from the top.

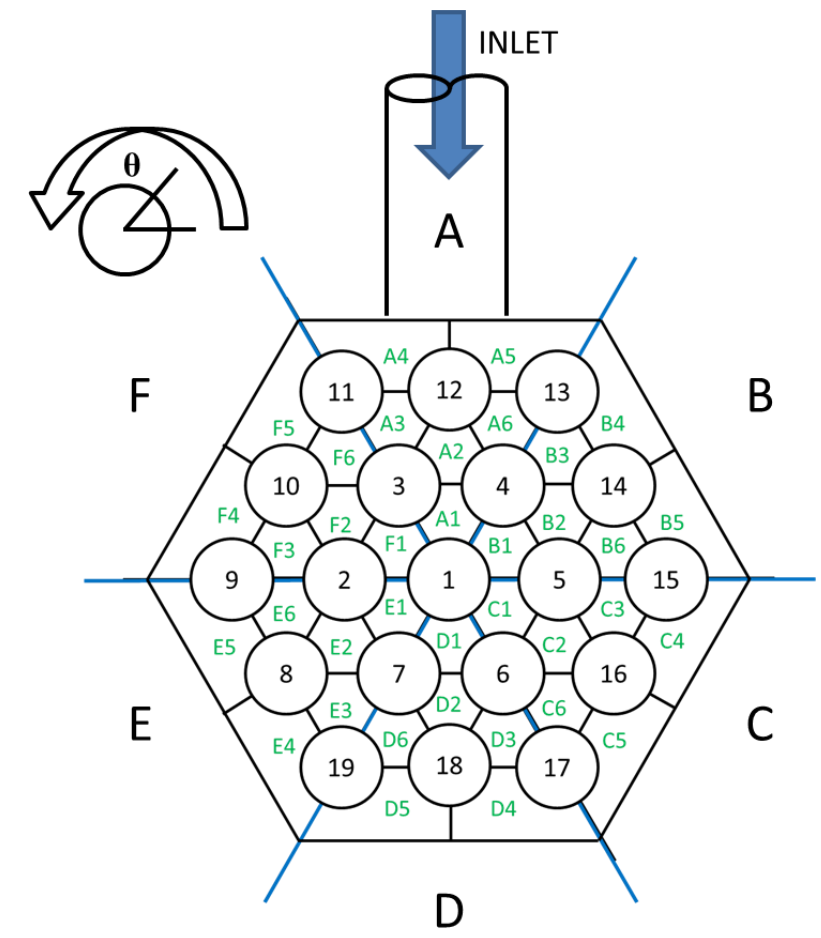

Figure 15 Sketch of a section of the new FPS for the NACIE-UP facility viewed from the top.

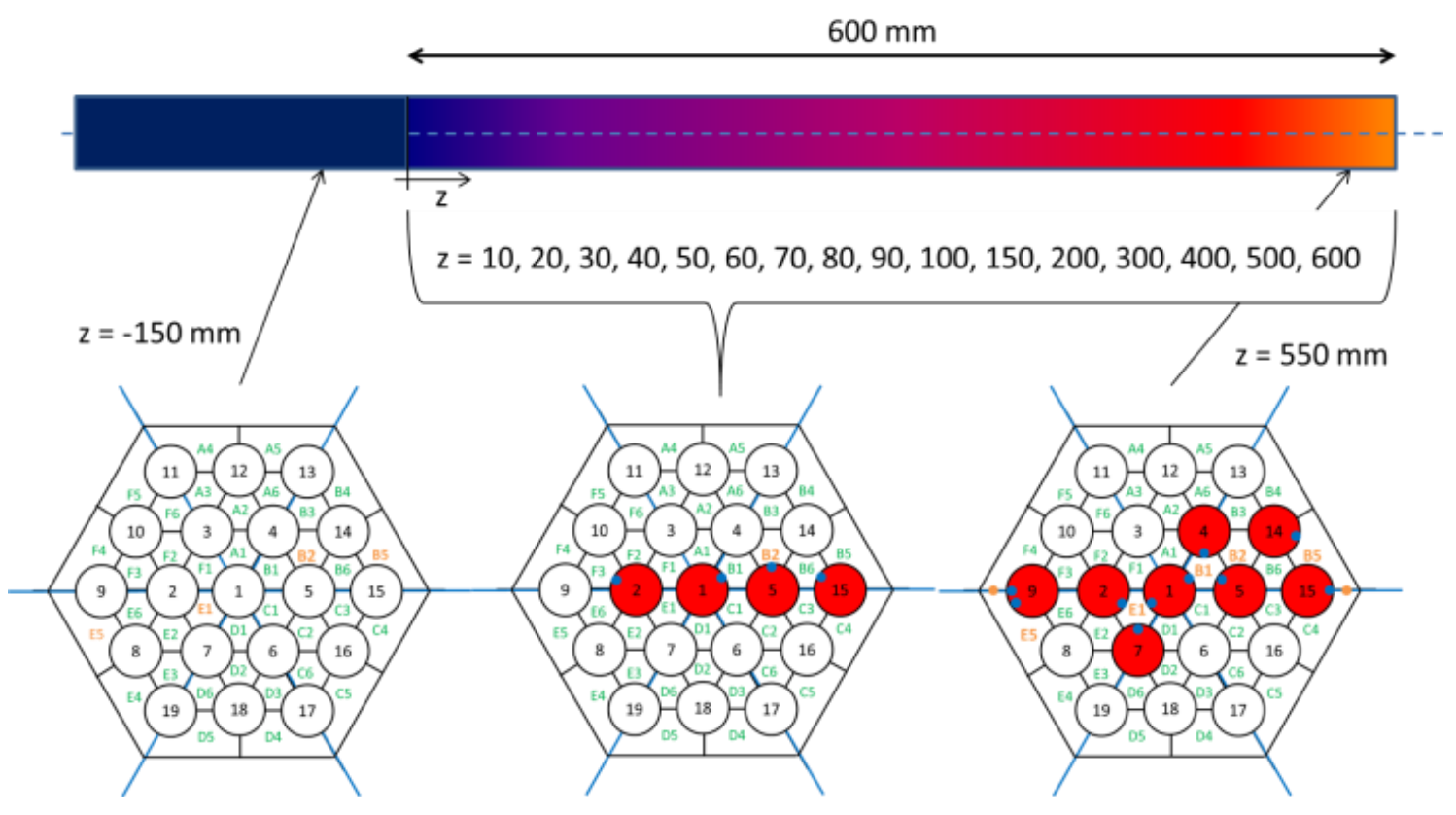

Figure 16 Overall pin bundle TC instrumentation 

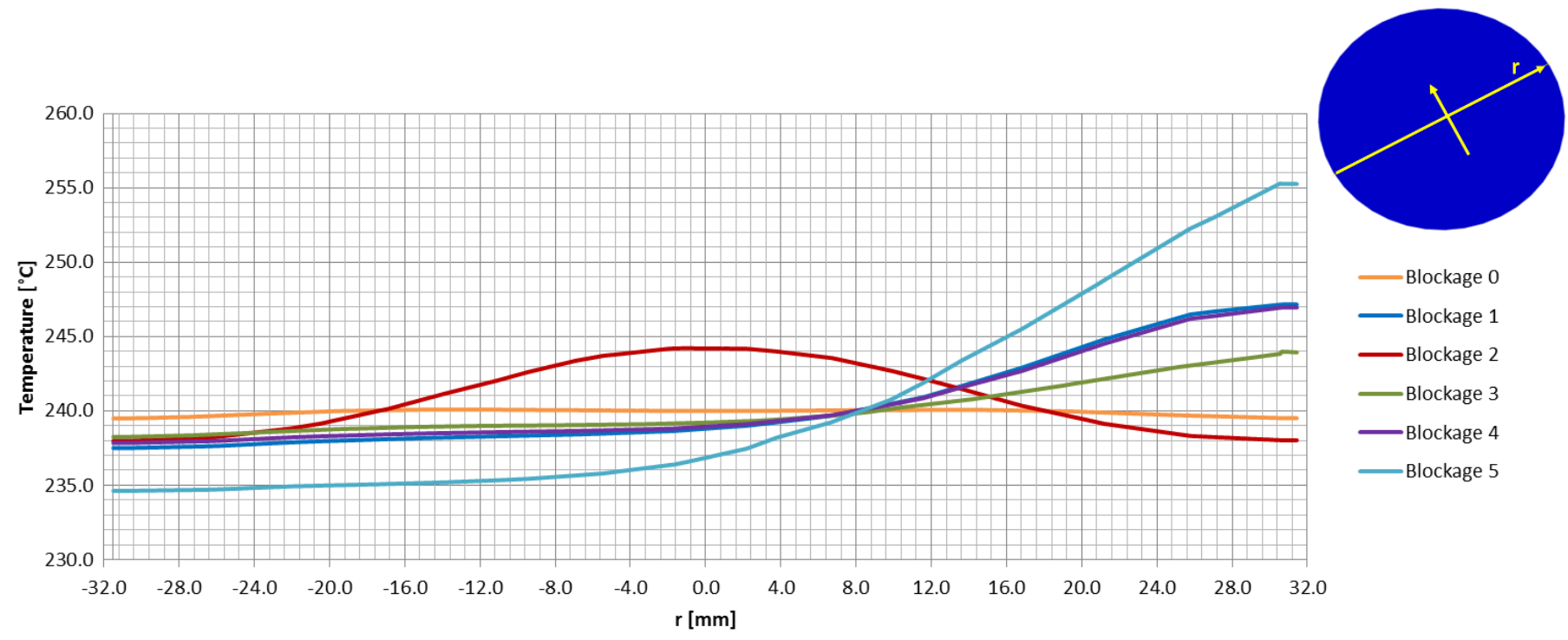

Figure 17 Temperature profile on a line placed at the outlet section of the model (top right sketch) for all the blockage types at 8 kg/s.
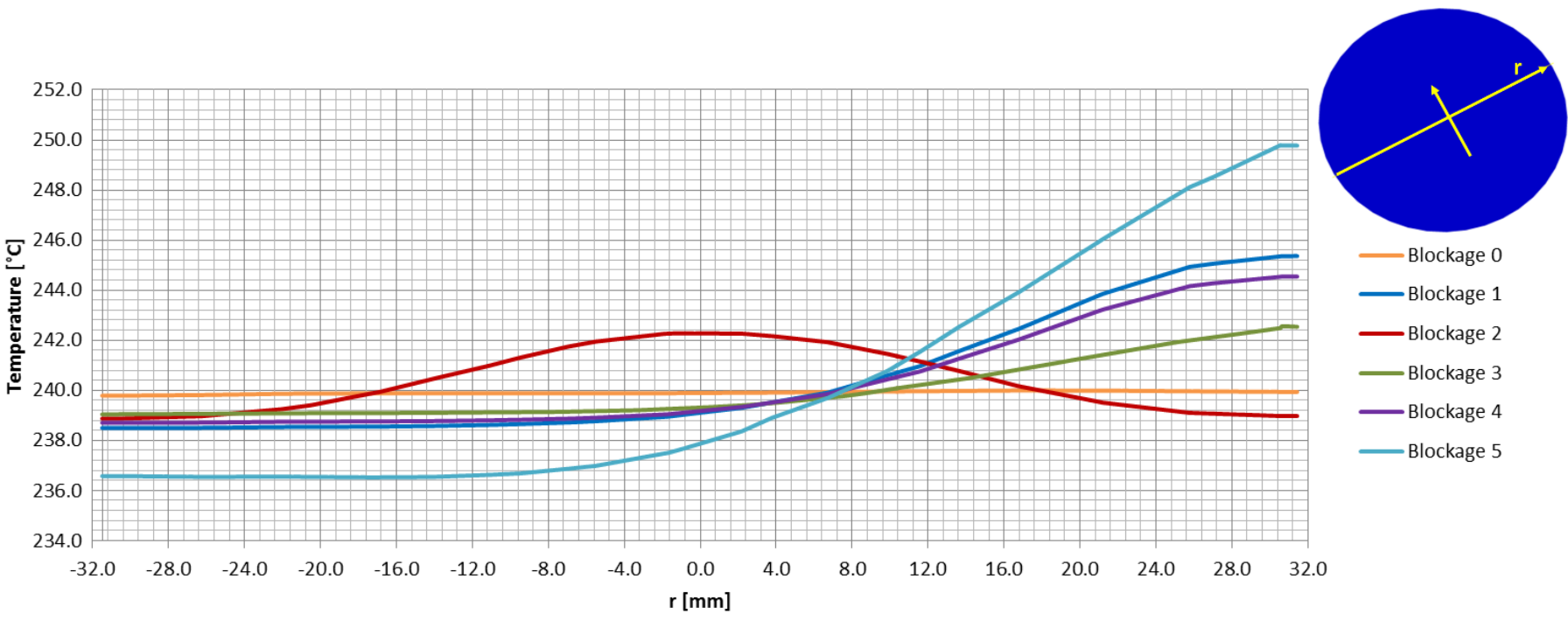

Figure 18 Temperature profile on a line placed at the outlet section of the model (top right sketch) for all the blockage types at $4 \mathrm{~kg} / \mathrm{s}$.

\section{Conclusions}

The present paper is focused on the CFD pre-test analysis of the 'Blocked' Fuel Pin bundle Simulator (BFPS) that will be installed into the NACIE-UP facility located at the ENEA Brasimone Research Center (Italy).

The BFPS test section will be installed into the existing NACIE-UP loop facility aiming to carry out suitable experiments to fully investigate the effects of different flow blockage regimes in a 19 fuel pin bundle providing experimental data in support of the ALFRED LFR DEMO development. In particular, the fuel pin bundle simulator (BFPS) cooled by lead bismuth eutectic (LBE), was conceived with a thermal power of about $250 \mathrm{~kW}$, a uniform wall heat flux up to $0.7 \mathrm{MW} / \mathrm{m}^{2}$. It consists of 19 electrical pins placed on a hexagonal lattice with a pitch to diameter ratio of 1.4 and a diameter of $10 \mathrm{~mm}$. 
Several blockage types (central, sector, side, corner) were simulated in the grid at the beginning of the active region with a subchannel velocity around $0.8 \mathrm{~m} / \mathrm{s}$, relevant for LFR. The aim of this pre-test calculation is to evidence basic phenomena and correctly place the instrumentation to capture relevant gradients.

Pre-test CFD analysis shows the same basic phenomena of previous studies on larger bundles (Di Piazza, 2014) and documented in literature (Schultheiss, 1987) (Kirsch, 1975). A strong local effect is evident behind the blockage area leading to an elongated vortex with a decrease of heat transfer between the coolant and the cladding. Consequently, in the region of influence behind the blockage, a temperature rise occurs both for the coolant and for the cladding. The extension of the region of influence is about $200 \mathrm{~mm}$ in the present case, but it scales with the extension of the blockage.

The local overheating is strictly dependent on the operational conditions. For the present case, safe conditions were chosen for the experimental FPS, i.e. $92 \mathrm{~kW}$ power with $40^{\circ} \mathrm{C}$ inlet-outlet temperature rise with an inlet temperature of $200^{\circ} \mathrm{C}$.

The local temperature increase due to the blockage is about $150^{\circ} \mathrm{C}$ for most of the cases in the present conditions.

The pre-test analysis confirms that blockage could be detected via properly placing the instrumentation, (wall-embedded and bulk thermocouples located along the active region, most of them in the region of influence of the blockage). Blockage could be detected placing TCs in the mixing region of the BFPS test section.

With this instrumentation, the BFPS experiment will provide unique local data on the FA blockage for HLM cooled configurations, by providing a database for code validation to extend the CFD blockage analysis to a full FA, and giving indications on the detection methods.

\section{Acknowledgments}

This work was performed in the framework of the H2020 SESAME project. This project has received funding from the Euratom research and training program 2014-2018 under grant agreement No 654935.

\section{References}

Cheng, X., Tak, N.I., 2006. CFD analysis of thermal-hydraulic behavior of heavy liquid metals in sub-channels. Nucl. Eng. Des. 236, 1874-1885.

Di Piazza, I., Magugliani F., Tarantino, M., Alemberti A., 2014. A CFD analysis of flow blockage phenomena in ALFRED LFR demo fuel assembly. Nucl. Eng. Des. 276, 202-215.

Di Piazza, I., Marinari, R., "CFD PRE-TEST ANALYSIS OF THE FUEL PIN BUNDLE SIMULATOR EXPERIMENT IN THE NACIE-UP HLM FACILITY" 16th International Topical Meeting on Nuclear Reactor Thermal - Hydraulics, NURETH-16, August 30-September 4, 2015, Hyatt Regency Chicago.

Doolaard, H.J., Shams, A., Roelofs, F., Van Tichelen, ,K., Keijers, S., De Ridder, J., Degroote, J., Vierendeels, J., Di Piazza, I., Marinari, R., Merzari, E., Obabko, A., Fischer, P., "CFD BENCHMARK FOR A HEAVY LIQUID METAL FUEL ASSEMBLY" 16th International Topical Meeting on Nuclear Reactor Thermal - Hydraulics, NURETH-16, August 30-September 4, 2015, Hyatt Regency, Chicago.

Greef, C.P., 1979. Temperature fluctuations: An assessment of their use in the detection of fast reactor coolant blockages. Nucl. Eng. Des. 52, 35-55.

Hae-Yong Jeon, Moon-Ghu Park, Seung-Hwan Seon, Jae-Ho Jeong, Effectiveness of Blockage Index for the Detection of Blockage in an SFR Subassembly, Transactions of the Korean Nuclear Society Spring Meeting, Jeju, Korea, May 29-30, 2014. 
Kirsch, D., 1975. Investigations on the flow and temperature distribution downstream of local coolant blockages in rod bundle subassemblies. Nucl. Eng. Des. 31, 266-279.

Maity, R.K., Velusamy, K., Selvaraj, P., Chellapandi, P., 2011. Computational fluid dynamic investigations of partial blockage detection by core-temperature monitoring system of a sodium cooled fast reactor. Nucl. Eng. Des. 241, 4994-5008.

Marinari, R., Ghionzoli, P.B., Forgione, N., Di Piazza, I., Tarantino, M., Magugliani, F., Alemberti, A., Borreani, W., CFD pre-test analysis and design of the NACIE-UP BFPS fuel pin bundle simulator, Proceedings of the 24th International Conference on Nuclear Engineering ICONE24 June 26-30, 2016, Charlotte, North Carolina, USA.

Menter, F. R., 1994. Two-Equation Eddy-Viscosity Turbulence Models for Engineering Applications. AIAA Journal 32(8), 1598-1605.

Naveen Raj, M., Velusamy, K., Maity, R. K., 2016. Thermal hydraulic investigations on porous blockage in a prototype sodium cooled fast reactor fuel pin bundle, Nucl. Eng. Des. 303, 88-108.

Nomoto, S., Yamamoto, H., Sekiguchi, Y., Tamura, S., 1980. Measurement of subassembly outlet coolant temperature in the JOYO experimental fast reactor. Nucl. Eng. Des. 62, 233-239.

Roelofs, F., Gopala, V.R., Chandra, L., Viellieber, M., Class, A., 2012. Simulating fuel assemblies with low resolution CFD approaches, Nucl. Eng. Des. 250, 548-559.

Schultheiss, G.F., 1987. On local blockage formation in sodium cooled reactors. Nucl. Eng. Des. 100, 427-433.

Seung-Hwan Seong, 2006. Establishment of the design requirements for a flow blockage detection system through a LES analysis of the temperature fluctuation in the upper plenum. Annals of Nuclear Energy 33, 62-70.

Weisemburger, A., Muller, G., Alemberti, A., Summary of HLM reactor materials ,LEADER FP7 WP6 report, 2013.

Wey, B.O.; Hughes, G.; Overton, R.S, Prediction of temperature fluctuations at the outlet of a blocked subassembly, Proceedings of the L.M.F.B.R. safety topical meeting, 1982. 